\title{
Obsessive and Compulsive Characteristics of Craving for Alcohol in Alcohol Abuse and Dependence
}

\author{
Jack G. Modell, Frederick B. Glaser, Louis Cyr, and James M. Mountz
}

\begin{abstract}
The purpose of this study was to quantify the extent to which subjective ratings of craving for alcohol in the alcohol-abusing or dependent person (herein, alcoholic) correlate with measurable and specific characteristics of obsessions and compulsions. The YaleBrown Obsessive Compulsive Scale modified to reflect obsessionality and compulsivity specifically related to heavy drinking (Y-BOCShd) was used for this purpose.

Highly significant correlations were found in the alcoholic population ( $n=62$ ) between subjectively rated craving for alcoholic beverages and several of the Y-BOCS-hd questions regarding alcohol-related thoughts and drinking behavior. Additionally, mean craving scores were considerably greater in the alcoholic population than in the matched control population $(n=62)$. The data suggest that craving shares specific features in common with the obsessions of obsessive-compulsive disorder and that the existence of craving is dependent on the presence of obsessive thoughts about drinking. Positive correlations between craving and measures of compulsive drinking behavior also were found; compulsive drinking behavior, however, may reflect the consequences of craving rather than a fundamental characteristic of craving itself. The data show that despite difficulties in defining the term craving, it is clearly a phenomenon that is experienced or endorsed by most alcoholic subjects and is not by most persons who do not abuse alcohol.

Key Words: Craving, Obsessions, Compulsions, Alcoholism.
\end{abstract}

$\mathbf{T}$ HE CONCEPT of craving for alcohol by individuals who abuse alcohol has long been shrouded in controversy and attended by debate over its precise definition. ${ }^{1-}$

${ }^{8}$ In the scientific literature, the term craving has been variously associated with physiologic, psychologic, and behavioral states; and despite the dictionary definition of craving as a strong desire, persons with alcohol or other drug problems use the word craving to mean any desire or urge, even a weak one, to use substances. ${ }^{9}$

Craving has also been likened to an obsession and compulsion, especially when associated with heavy drinking. ${ }^{10}$ Obsessions and compulsions are seen most prominently in obsessive-compulsive disorder (OCD). In this

From the Department of Psychiatry (J.G.M.), Department of Biostatistics and Biomathematics (L.C.), and Department of Radiology (J.M.M.), University of Alabama, Birmingham, Alabama; and the Department of Psychiatry and Substance Abuse Center (F.B.G.), University of Michigan, Ann Arbor, Michigan.

Received for publication November 20, 1991; accepted November 27, 1991

This work was supported by a grant from the National Institute on Alcohol Abuse and Alcoholism (5-P50 AA07378).

Reprint requests: Jack G. Modell, M.D., Department of Psychiatry, University of Alabama, Smolian Psychiatric Clinic, Room 403, UAB Station, Birmingham, AL 35294-0018.

Copyright $(1992$ by The Research Society on Alcoholism. disorder, the affected individual usually describes recurrent and persistent thoughts (obsessions) that impel the performance of a behavior (compulsion) that is recognized by the individual as maladaptive or potentially destructive; we have noted that many of our patients who abuse alcohol describe craving in a similar way. Additionally, cue-exposure response-prevention methods of treatment similar to those used for the treatment of $O C D$ also effectively reduce the desire to drink and facilitate abstention in alcohol-addicted individuals. ${ }^{11}$

The purpose of the current study was to amplify the definition of craving by quantifying the extent to which subjective ratings of craving for alcohol in individuals satisfying DSM-III-R criteria for alcohol abuse or dependence [herein, alcoholic(s)] correlate with measurable and specific characteristics of obsessions and compulsions as occur in OCD. We expected that because craving is usually considered more of a cognitive than a behavioral phenomenon, craving would be more highly correlated with the characteristics of an obsession than those of a compulsion.

\section{METHODS}

The experimental population was composed of 62 subjects ( 47 men, 15 women; mean age 39, range 22 to 67 years) satisfying DSM III-R criteria for alcohol abuse or dependence and no other axis I disorder; experimental subjects were in treatment for alcohol abuse at the University of Michigan or University of Alabama Psychiatric Outpatient Departments, or the Ann Arbor Veterans Administration Hospital (inpatient). The last drink of alcohol was 2 days to 4 weeks before study induction for all alcoholic subjects. The control population consisted of 62 subjects ( 47 men, 15 women; mean age 39 years, range 24 to 69 ) who did not fulfill criteria for alcohol abuse, dependence, or any other DSM III-R axis I disorder. The groups were age, sex, and education matched.

Each experimental and control subject was initially asked, "On a scale of 0 to 10 -where 0 is not at all, and 10 is the most imaginable-how much do you crave an alcoholic beverage when you've gone without a drink for 1-2 days?" The term "crave" was intentionally not defined. The Yale-Brown Obsessive Compulsive Scale ${ }^{12,13}$ modified to reflect obsessionality and compulsivity specifically related to heavy drinking rather than to obsessions and compulsions generally (Y-BOCS-hd) ${ }^{14}$ was then administered to all study subjects by one of the investigators (J.G.M.). Questions 1 through 5 of the Y-BOCS-hd comprise the obsessionality subscale and primarily reflect drinking-related thought; questions 6 through 10 of the Y-BOCS-hd comprise the compulsivity subscale and primarily reflect drinking-related behavior.

Correlations between subjectively rated craving and each of the ten Y-BOCS-hd questions as well as the sums of questions 1 through 5, 6 through 10 , and 1 through 10 were obtained by Spearman's rank correlation coefficient. The Mann-Whitney-Wilcoxon test was employed for comparison of subjectively rated craving between the alcoholic subjects and normal controls. Nonparametric statistical procedures were used 
because of the ordinal nature of the Y-BOCS-hd data. We adopted $\alpha<$ 0.001 as our level of significance for all comparisons to provide a conservative interpretation of results.

\section{RESULTS}

Significant positive correlation coefficients were found in the alcoholic population between subjectively rated craving for alcoholic beverages and the Y-BOCS-hd questions on the obsessionality subscale concerning the amount of time occupied by alcohol-related thoughts (question $1, r=0.45$ ), the amount of resistance mounted against alcohol-related thoughts (question $4, r=0.45$ ), and degree of control over alcohol-related thoughts (question $5, r=0.52$ ). On the compulsivity subscale, significant positive correlation coefficients were found in the alcoholic population between craving and the Y-BOCS-hd questions concerning the usual quantity and frequency of alcohol consumption (question $6, r=0.50$ ), the amount of distress that would be experienced if the individual were prevented from drinking when desired (question 8, $r=$ 0.48 ), and the perceived degree of control over consumption of alcoholic beverages (question 10,r=0.48). The correlation between craving and the Y-BOCS-hd was strengthened by use of a stepwise regression procedure that incorporated Y-BOCS-hd questions 6 and $8(r=0.62$, $p<0.001)$. Significant correlations also were found between subjectively rated craving and the Y-BOCS-hd obsessionality subscale sum $(r=0.53)$, compulsivity subscale sum $(r=0.59)$, and full-scale sum $(r=0.58)$. Conversely, craving in the alcoholic group was not significantly associated with the degree of interference in function or distress caused by alcohol-related thoughts, the degree of interference in function caused by drinking, or the degree of resistance mounted against drinking.

In the nonalcoholic controls, the only individual item on the Y-BOCS-hd to which craving was significantly correlated was question 6 pertaining to the usual quantity and frequency of alcohol consumption $(r=0.50)$. Significant correlations in the control group were also found between subjectively rated craving and Y-BOCS-hd compulsivity subscale sum $(r=0.50)$ and full-scale sum $(r=$ 0.61 ). There were no significant correlations in the control group between craving and any individual question on the obsessionality subscale; additionally, the responses to the questions on the obsessionality subscale in this group were tightly clustered around zero ( $\overline{\mathrm{x}}=0.01-0.05)$.

Reported levels of craving in the alcoholic population averaged 6.5 (range $0-10, \mathrm{SD}=3.0$ ). In contrast, craving in the nonalcoholic controls was minimal $(\overline{\mathrm{x}}=0.24, \mathrm{SD}$ $=0.64$, range $0-3$ ), and only three normal controls rated craving greater than 1.0. These differences in craving between the alcoholics and normal controls were highly significant $(I Z=9.2, p<0.001)$. Sample size limitations did not permit valid assessment of age or sex differences in the outcome measures.

\section{DISCUSSION}

The positive correlations in the alcoholic group between reported levels of craving for alcoholic beverages and the obsessional subscale items support our hypothesis that craving shares specific features in common with the obsessions of OCD. The very low magnitude of reported craving and essentially flat, zero-magnitude responses on the obsessionality subscale questions in the normal controls is consistent with the view that the existence of craving is dependent on the presence of obsessive thoughts about drinking.

The nearly equal correlations in the alcoholic group between craving and the questions on the Y-BOCS-hd compulsivity subscale and those on the Y-BOCS-hd obsessionality subscale is contrary to our initial expectation that craving would be more highly correlated with the characteristics of obsessions than compulsions. The most probable explanation for this finding is that the positive correlations with actual drinking behavior (compulsivity subscale) reflect the consequences of craving rather than a fundamental characteristic of craving itself; it might, for example, be expected that the more one "craves" alcohol, the greater the amount of alcohol that would be consumed, the greater the distress that would be felt if drinking were prevented, and the less control the individual would have over alcohol consumption. Alternatively, actual drinking behavior might be used by the individual as a post hoc estimator of craving.

Although this study revealed statistically significant correlations in the alcoholic group between craving and the Y-BOCS-hd subscales and some of the individual questions, the strengths of these correlations were somewhat modest. The data could, therefore, be interpreted as indicating that craving shares relatively little in common with the obsessive and compulsive characteristics of OCD, despite apparent similarities between craving and "obsessions" and "compulsions" as defined in common parlance. Such difficulties in precisely defining craving have been noted in the past and used to argue for abandonment of this term in substance abuse research. ${ }^{9}$ Nevertheless, a comparison of the mean craving scores between the control and alcoholic populations clearly demonstrates that despite the difficulties in defining craving, it is clearly a phenomenon that is experienced or endorsed by most alcoholic subjects, and is not experienced by most individuals who do not abuse alcohol. This finding argues for the importance of further research into the clinical significance of the concept of craving. The use of subjectively rated craving as an independent variable in carefully conducted treatment-outcome studies, especially those in which ratings of craving are paired with other measures of drinking-related thought and behavior, seems well-suited for this purpose.

\section{REFERENCES}

1. Jellinek EM, Isbell $\mathrm{H}$, Lundquist $\mathrm{G}$, et al.: The "craving" for alcohol: a symposium by members of the WHO expert committees on mental health and on alcohol. Q J Stud Alcohol 16:34-66, 1955 
2. Kozlowski LT, Wilkinson DA: Use and misuse of the concept of craving by alcohol, tobacco, and drug researchers. Br J Addict 82:31-36, 1987

3. Ludwig AM, Wikler A: "Craving" and relapse to drink. Q J Stud Alcohol 35:108-130, 1974

4. Maisto SA, Schefft BK: The constructs of craving for alcohol and loss of control drinking: help or hindrance to research. Addict Behav 2:207-217, 1977

5. Rankin H, Hodgson R, Stockwell $T$ : The concept of craving and its measurement. Behav Res Ther 17:389-396, 1979 1981

6. Rankin H: Craving competition (editorial). Br J Addict 76:1-2,

7. Rankin H: Some comments on the 'use and misuse of the concept of craving' by Kozlowski \& Wilkinson: craving is more than a wooly scientific concept. Br J Addict 82:981, 1987

8. Shiffman S, Hughes JR, West R, Marlatt GA, Stockwell T: Comments on Kozlowski \& Wilkinson's 'use and misuse of the concept of craving by alcohol, tobacco, and drug researchers.' Br J Addict 82:3746,1987
9. Kozlowski LT, Mann RE, Wilkinson DA, Poulos CX: "Cravings" are ambiguous: ask about urges or desires. Addict Behav 14:443-445, 1989

10. Caetano R: Alcohol dependence and the need to drink: a compulsion? Psychol Med 15:463-469, 1985

11. Rankin H, Hodgson R, Stockwell T: Cue exposure and response prevention with alcoholics: a controlled trial. Behav Res Ther 21:435446,1983

12. Goodman WK, Price LH, Rasmussen SA, et al.: The Yale-Brown Obsessive Compulsive Scale: II. Validity. Arch Gen Psychiatry 46:10121016,1989

13. Goodman WK, Price LH, Rasmussen SA, et al.: The Yale-Brown Obsessive Compulsive Scale: 1 . Development, use, and reliability. Arch Gen Psychiatry 46:1006-1011, 1989

14. Modell JG, Glaser FB, Mountz JM, Schmaltz S, Cyr L: Obsessive and compulsive characteristics of alcohol abuse and dependence: Quantification by a newly developed questionnaire. Alcohol Clin Exp Res $16: 266-271,1992$ 